\title{
Towards fairer borders: Alleviating global inequality of opportunity
}

\section{Magnus Skytterholm Egan}

Institute of Philosophy, UiT - The Arctic University of Norway, Tromsø, Norway, magnus.egan@uit.no

DOI: http://dx.doi.org/10.5324/eip.v12i2.2421

(cc) BY This is an open access article distributed under the terms of the Creative Commons Attribution 4.0 International License, which permits unrestricted use, distribution, and reproduction in any medium, provided the original author and source are credited.

Current admission criteria for migrants tend to favor those who are well to do, able bodied, and well qualified. This leads to migration patterns that exacerbate global inequalities. In this article, I argue that we should alter admission criteria in order to alleviate some of the negative effects of global inequality of opportunity. In support of this argument, I discuss two global justice theories that are central to borders and migration, specifically a cosmopolitan position that argues for more open borders and a nationalist position that emphasizes the importance of states being in control of their borders. In particular, I address David Miller's objections to using open borders to remedy global inequality of opportunity. The argument I present agrees with the benefits of a conception of justice that allows for degrees of partiality and a state's right to control its borders. However, I argue that the roles of Western states in particular in perpetuating global inequality of opportunity lead to moral demands, which can in part be met by fairer migration. Finally, I consider what kind of criteria fairer immigration should take into account. The system I propose would rank migrants based on their individual access to opportunities, how little their emigration would affect the opportunities in the country they are emigrating from, and to what extent it might improve the opportunities in the country they are moving to.

Keywords: borders, equality of opportunity, global justice, migration

\section{Introduction: Why is migration relevant to questions of global equality of opportunity?}

Most normative approaches to political philosophy proceed from the generally held assumption that all human beings are of equal worth. However, the kinds of implications this assumption has for how one should actually treat people are hotly disputed. For although most people agree that all human beings are equally valuable, political institutions, private companies, and the international community treat people differently based on many different criteria, such as religious, economic, linguistic, or national distinctions. One of the ways in which people are treated differently is in regards to whether they are allowed into a country or not. An important task for political philosophers is to consider which kinds of differentiated treatment are morally justified and which are not. 
When it comes to migration and questions of global justice, a useful distinction can be made between nationalist and cosmopolitan positions. Whereby the former emphasizes the reasons states are justified in prioritizing their own citizens over foreigners and argues for controlled migration, the latter emphasizes the illegitimacy of many migration restrictions and argues for open borders or more freedom of movement. ${ }^{1}$ In terms of immigration, they disagree over whether selective immigration regulations are normatively valid, and in which situations and to what extent this might be the case. Broadly put, cosmopolitans argue that the demands of global justice are so strong that a state is only justified in implementing limited migration restrictions, while nationalists argue that the global justice demands are weaker and that it is in everyone's basic interest that states have comprehensive control over their migration restrictions. In this article, I am specifically concerned with voluntary migration, as opposed to forced displacement ${ }^{2}$, and with one of the arguments often put forth for the cosmopolitan position, namely global equality of opportunity. I will be using the equality of opportunity argument in order to defend a fairer migration model. This model evaluates whether migration regulations are justified not merely based on the impact a migrant might have on the country they are moving to, but also considers the effect on the country they are leaving, and the improvement in opportunities for the individual migrant.

Before considering why migration might be relevant to questions of global equality of opportunity, I will first describe what such an equality might consist of. Perhaps the most prominent exponent of the cosmopolitan position on migration is Joseph Carens, who argues that open borders are necessary partly in order to accomplish equality of opportunity. ${ }^{3}$ Carens describes the equality of opportunity argument as follows:

Within democratic states we all recognize, at least in principle, that access to social positions should be determined by an individual's actual talents and effort and not limited on the basis of birth-related characteristics such as class, race, or gender that are not relevant to the capacity to perform well in the position. This ideal of equal opportunity is intimately linked to the view that all human beings are of equal moral worth, that there are no natural hierarchies of birth that entitle people to advantageous social positions. But you have to be able to move to where the opportunities are in order to take advantage of them. So, freedom of movement is an essential prerequisite for equality of opportunity $(2013,227-228)$.

Simply put, this is an argument for open borders. Moreover, it proceeds from the premise that the stark divide between nations in access to opportunities is vast; that where people are born is a matter of chance, and that this disparity is therefore fundamentally unfair and should be rectified. In other words, it would seem that if we believe all people are equally valuable, we should provide them with similar access to opportunities. This argument is consistent with economic and social differences between people, often referred to as inequalities in outcomes, and is based on the intuitively appealing idea that people should have an equal chance to attain favored social positions. However, it is still necessary to specify what these opportunities consist in, and which social goods equality of opportunity relies upon. As Carens puts it, "[a] closely related point is that a commitment to equal worth entails some commitment to economic, social, and political equality, partly as a means of realizing equal freedom and equal opportunity and partly as a desirable end in itself' (2013, p. 228). I therefore initially consider equality of opportunity broadly to mean access to 
social positions, and that this access relies on the availability of social goods such as access to health care, education, and the job market. ${ }^{4}$ As we will get back to below, however, how one chooses a particular metric, and whether one can make a global metric of opportunities, is hotly contested.

This is quite a broad definition to proceed from, as it hardly seems unreasonable to make the point that current global differences in opportunities are unfair and something we should remedy. However, this perspective raises questions of whether the goal of just policies should be the complete elimination of differences, the mitigation of significant differences, and what complete equality might look like. Furthermore, how far is fairer migration supposed to move us towards this goal? The answers to these questions lie beyond the scope of this article, and I am not advocating an ideal situation. Rather, I am pointing to current unfairness in migration practices and making some suggestions as to how this unfairness could be mitigated. Thus far, I can be seen as following Miller, whose stated aim was "to sketch the outlines of a legitimate and justifiable immigration policy for a democratic state in the world that we actually inhabit, replete with inequalities and injustices." (2016b: 2) In short, I am moving from broadly uncontroversial views on global differences in opportunity, to some potentially more controversial suggestions about how these differences can be reduced. I begin by addressing two objections to using equality of opportunity as an argument for open borders, specifically (1) that it is problematic to compare opportunities between different societies, and (2) that opportunities are largely the result of states' domestic policies. In light of this discussion, I then consider why altered migration regulations, rather than open borders, might contribute towards global equality of opportunity, before finally presenting a sketch of migration regulations that can contribute towards this goal.

\section{Nationalist objections to global equality of opportunity}

Before discussing how this type of equality of opportunity is relevant to questions of migration, I will consider some objections to the argument that in order to have global equality of opportunity, we need open borders. In Strangers in Our Midst, David Miller presents a comprehensive and convincing defense of the nationalist position, and argues against the relevance of global equality of opportunity for evaluating whether migration restrictions are just or not. The argument I propose must therefore be able to meet his objections. Miller attacks the equality of opportunity argument for open borders by arguing that: (1) there are problems in evaluating opportunities across cultures, (2) the level of opportunities are largely the result of domestic politics in states, and (3) there is a possibility of a brain drain from the countries of origin. The point about the brain drain will be addressed in the next section. Before doing so, I will comment on Miller's other two points in particular and the nationalist position in general.

\section{One cannot compare opportunities across cultures}

First, let us consider the suggestion that evaluating opportunities across cultures poses problems. As Miller puts it: "There is no agreed metric that can be applied to rank the sets because how particular opportunities are to be valued relative to one 
another will depend upon the local culture" (Miller 2016a: 46). It should be stressed that Miller does not think this applies to basic human rights, and he draws a strict division between basic rights and equality of opportunity. The differences which he does not think can be used as a normative basis when it comes to alleviating global inequality of opportunity through migration, are differences relating to things such as "to get an education, to enter the job market, and to make money" (Miller 2016a: 44). While the merits of such a differentiation might be debated, let us take it for granted for the purpose of the present discussion.

Miller's argument does not claim that it is difficult to measure the differences between cultures, but rather that these kinds of metrics out of necessity prioritize one particular cultural vantage point. Different nations and cultures prefer different kinds of opportunities; therefore a comparison between them is not relevant to questions of global justice and migration. Another formulation of this argument is put forward by Gillian Brock, who argues,

Either we must try to articulate a version of equality of opportunity that mentions particular social positions that are favored, and opportunities to achieve these are equalized, or we allow much cultural variation on what counts as a favored social position, and the standards of living or levels of well-being that they enable are to be equalized (2015: 29).

Put simply, the problem is that either the favored social positions and standards of well-being are too broad, and therefore cannot be useful as a comparative metric, or the metric favors some particular cultural standard over others.

Now, while it is certainly the case that various countries prioritize different sectors, such as health, education, and the job market, it does not necessarily follow that their ability to prioritize these sectors is the result of national priorities. And, if their ability to provide opportunities is significantly affected by outside factors, the fact that they might prioritize various sectors does not necessarily remove the moral culpability for creating this disparity. We consider this more closely in the next section, so let us first turn to the contention that it is in fact necessarily parochial to rank these opportunities up against each other.

It is clear that different cultures have variations in preferences. However, that does not mean that no significant overlap exists, and that this overlap might not be meaningful in discussions about variations in opportunities. For while differences in preferences might vary, it is a much stronger claim that they vary to such a degree that comparisons between them are not valuable in moral discussions about differences in equality of opportunity. After all, one would be hard pressed to make the case that some cultures prefer high unemployment and poor access to decent health care. Indeed, just because states have internal variations with respect to preferences, we do not consider this a reason to abandon an attempt at overall considerations of justice and opportunities in the domestic setting. And while the variation is undoubtedly greater in the international setting, the mere fact of difference does not make comparisons invalid as foundations for normative evaluations. As Darrel Moellendorf argues, Brock's cultural variation challenge can be met "if there were an account of the goods - for which opportunities should be equalized - that is both free-standing, that does not derive simply from the cultural understandings of a particular culture, and sufficiently sensitive to empirical matters as to capture real differences of opportunity" (2006: 309). In short, we would need a 
sufficiently general and empirically sensitive metric in order to make valuable comparisons.

Furthermore, access to education, the job market and decent health care certainly seem to be likely contenders as aspects of society that are globally valued. As Eszter Kollar points out, "some global egalitarians have responded [to problems of global value pluralism] by proposing a core set of goods that should be seen as all-purpose means in global political life" (2016: 4). So, while the content to be included in such an evaluative standard will be hotly disputed, the fact of value pluralism does not mean that a variety of meaningful evaluative standards cannot be found.

There are also various ways in which one can approach the problem of deciding on an overall metric. One might concede that it is difficult to agree on such a global metric, and yet believe that meaningful comparisons can be made in the case of evaluating the opportunities of individual migrants. As I propose below, a measure of opportunities for individual migrants might take into account their access to education, the job market and health care in both the country they are moving from and the receiving country. So, while we might struggle to create an overall metric for all people, we can likely say something meaningful about specific migrants' access to opportunities and how these may or may not be improved.

In short, I do not make the claim that we can create some kind of overall objective metric by which to consider all opportunities and global justice. I am merely contending that some relative comparisons are normatively meaningful, in particular when it comes to judging potential migrants. Furthermore, while all people probably cannot agree on a standard system of differentiation, they can surely provide some facts as a basis for reasonable democratic deliberation of responsibility. Perhaps a general description of variations in opportunities and obligations that stem from them could be put forth. After all, we seldom demand empirical exactitude on domestic considerations of justice from philosophers, so why should this be the case in the international setting?

\section{One should not compare opportunities across cultures}

Another and perhaps more central aspect of Miller's objection to the possibility of evaluating differences is that it is not fair to use these varying opportunities as a measure, since they follow from the self-determination of the countries in question. In other words, these differences are largely the results of different priorities made by different states, and therefore not relevant for the current discussion. As Kollar formulates this nationalist argument, "[g]lobal equality of opportunity wrongly neglects the normative relevance of national self-determination that inevitably produces different opportunity sets for different nationalities. [...] It is not that we cannot compare, but that we should not compare opportunities across borders" (2016: 4). Therefore, we must consider the question of how these differences in opportunities come about, and make the case for why these differences are in fact relevant for our present discussion.

As most people would readily agree, the differences in opportunities across borders are vast, in particular between countries in the Global North and the Global South. However, there is a lot of disagreement about whether, and to what degree, these differences are the outcome of legitimate national priorities or due to other causes. A nationalist position might state that these differences in opportunities are 
not normatively relevant to questions of admission policies, insofar as they are the result of domestic political decisions for which the nation can be held responsible. Higgins describes this nationalist argument as follows:

If the global poor are understood merely as needy strangers whose poverty is causally unrelated to the affluence of the Global North, then choosing admission policies in a way that is responsive to their interests appears, wrongly, to be a matter of charity (2013: 30).

An initial objection to the nationalist position might question why migrants should be held responsible for the domestic policies in their home countries. Indeed, most of these kinds of decisions are taken before and without most individuals' explicit blessing. Why should the opportunities in a country therefore count for anything in evaluating whether migration restrictions are justified? A nationalist will likely respond that the relevant question here is who bears the responsibility to fix global disparities in opportunities. How can one make the case that a prosperous country should alleviate global differences, if they are not responsible for bringing these differences about? While I do think this objection has merit, the nationalist position should nevertheless be addressed.

It is clearly correct to state that the level of opportunities is to a certain extent the result of domestic policy. However, this is hardly the whole story. The level of opportunities in countries is also influenced by other countries' foreign policy, trade policy, and the international political system to a considerable degree. Even when it is difficult to show exactly how disadvantages come about, this still does not mean that the causes lack normative validity or do not place moral demands on us. The point is that global inequalities generally have complex causes, and are not merely due to national priorities. As Kollar (2016: 5) says, "Those differences that are not the outcome of legitimate national priorities, instead, should be judged as unjust global inequalities of opportunity to be properly mitigated from life-prospects."

One way of explicating this unequal relationship can be found in the work of Thomas Pogge. He points out how certain features of the international system systematically disadvantage the ability of other countries to fulfill the human rights of their citizens. For instance, Pogge (2001: 20) describes the "international borrowing privilege" and the "international resources privilege." The former in part "facilitates borrowing by destructive governments" and "imposes upon democratic successor regimes the often huge debts of their corrupt predecessors" (Pogge 2001: 20). "Resources privilege" allows the group in power in a country to be considered the legitimate owners of that country's resources, whether their citizens are represented or repressed. In short, Pogge explicates how these privileges maintain an unfair international system, which benefits the interests of some in richer and more influential states over others. If we take this to be the case, it seems clear that the vast differences in opportunities between states are not the result of legitimate national priorities, but rather the result of unfair trading practices. In other words, regardless of how some countries might decide to prioritize, the vast differences in global opportunities will remain.

Another point pertains to aspects of the international financial system, which allows for vast transfers of wealth from poor to rich countries. A recently published report by Global Financial Integrity and the Center for Applied Research at the Norwegian School of Economics shows how the financial resources that flow from 
poor to rich countries are far greater than those that flow in the opposite direction, and that the international financial system allows for this reverse distributive effect:

In 2012, the last year of recorded data, developing countries received a total of $\$ 1.3 \mathrm{tn}$, including all aid, investment, and income from abroad. But that same year some \$3.3tn flowed out of them. In other words, developing countries sent \$2tn more to the rest of the world than they received. If we look at all years since 1980, these net outflows add up to an eye-popping total of \$16.3tn - that's how much money has been drained out of the global south over the past few decades (Hickel 2017).

In sum, it can be argued that there are many aspects of the international economic, political and financial system that contribute to countries' financial ability and political stability. Furthermore, since the level of opportunity in a country relies directly upon the government's ability to deliver decent health care, education, and job markets, a grossly unfair international system that perpetuates these differences seems particularly relevant when considering states' responsibilities to alleviate them.

\section{Why might migration be relevant to alleviate global inequality of opportunity?}

Even allowing for the arguments that some states benefit from an unjust global system and contribute to perpetuating global inequality, some might ask why this inequality is relevant to questions of migration and admission policies. Some people might indeed say that all I have done is show that some trade policies are unjust, that these affect the levels of opportunities, and that this is something that should be remedied. However, why should the solution to this problem have anything to do with migration? Responding to this challenge requires answering the question of how open borders, or increased migration, might contribute to alleviating this inequality. One way to respond is to follow Carens, who states, "Freedom of movement would contribute to a reduction of existing political, social, and economic inequalities. There are millions of people in poor states today who long for the freedom of economic opportunity they could find in Europe or North-America" (2013: 228). But as Miller and Higgins, among others, have pointed out, the idea that open borders would significantly reduce global inequalities does not mesh well with how migration actually occurs. Indeed, as it is only those who can afford to who can travel, it has been argued that open borders would not contribute towards alleviating global inequality of opportunity, and might even negatively affect it. ${ }^{5}$ According to Higgins, the open border position relies "on an idealized conception of the typical poor migrant's social wherewithal, as well as on an explanatorily nationalist understanding of the causes of severe poverty" (2013: 64). In other words, this position builds on a wrongly held belief that the causes of severe poverty are local, and that people lacking opportunities and basic rights have the social and financial resources to migrate. As Higgins puts it, "the structural causes of social disadvantage in virtue of which persons are vulnerable to severe poverty are not themselves addressed by changing potential migrants' location of residence" (2013: 64).

Since open borders might not be a solution to the problem of alleviating global inequalities of opportunity, why should we care about migration at all in connection with global inequalities? Here we must distinguish between arguments for open 
borders and arguments for fairer migration. Although the former might not improve global equality of opportunity, it seems more likely that fairer migration, which takes into account global inequality of opportunity, would do so. Nevertheless, some might argue that given the effort it would take to alter unfair admission practices, we should instead focus on remedying an unfair international system. As Pogge argues, "With the political effort it would take to pressure some Western government to admit an extra hundred needy foreigners, we could alternatively pressure this same government to allocate a few extra million dollars to global poverty eradication" (1997: 17). In other words, rather than making the difficult point that we should admit people according to fairer migration criteria, our efforts are better spent arguing for more practical measures to reduce extreme poverty.

One can respond to this objection in several ways: first, by pointing out that arguing for fairer migration will also indirectly highlight the inequalities inherent in the global system. In other words, a normative reconstruction of our admission criteria might draw attention to global inequalities, which most definitely need addressing. Second, one might show how some unfair admission criteria actually contribute to, indeed are perhaps even integral to, the unjust international order. So, whereas poverty eradication might indeed give more of a return for each dollar spent, unfair admission criteria can be shown to play an integral role in a globally unequal system that contributes to extreme poverty. Third, one might contend that this is not a zero sum game, and that the more arguments that are raised concerning global inequalities and the systems that perpetuate them, the better.

In short, I argue that because many affluent countries are apparently unwilling to rectify global inequalities, and in some cases even actively perpetuate them, these countries have a moral obligation to admit migrants on fair terms. I am not claiming that this is the ideal solution to the problem of global inequality of opportunities, merely that it is relevant to questions of justice and migration. While these arguably justifiable demands for fairer migration might not be met either, it is still important to point them out.

Finally, some people might criticize both open borders and my suggestions for fairer migration criteria by pointing out that states have a right to control their borders, and that this right is needed, and normatively justified, in order to uphold the basic functions of a liberal democratic state. Miller states that liberal democracies have a right to control their borders due to concerns over "self-determination, the functioning of democracy, and population size" (2016a: 75). So there are good reasons to assume that some selective admission criteria are necessary to facilitate the proper functioning of a working democracy. However, even though a state might be justified in limiting and controlling immigration, the migration restrictions that a state implements can still be evaluated as to whether they are conducive to global equality of opportunity or not.

The question then arises of what type of admission criteria would contribute to global equality of opportunity. If states are justified in controlling immigration, and open borders are not conducive to global equality, what kind of immigration regulations would alleviate global inequalities of opportunity? Furthermore, how can we practically evaluate whether states' immigration regulations are appropriate to achieving such a goal? As previously discussed, a universally accepted metric of global equality of opportunity is unlikely, and states are more likely to have readily available information on the opportunities of specific migrants seeking admittance. 
Specifically, countries are more likely to be able to deliberate on relative differences in opportunities between sending and receiving countries than the effect migration regulations might have on overall global opportunities. The most readily available data for countries to consider is the impact a potential migrant has on the country they come from, the country they are moving to, and the effect of the move on the individual migrants. These factors could work as proxies, allowing migration regulations overall to be conducive to global equality of opportunity. Put simply, the moral reasons for countries to alter their admission criteria are the vast global disparities in opportunities, and the practical way to alter the admission criteria is based on the data readily available to the countries in question. In the following, I present an outline for what such fairer admission criteria would look like.

\section{What kind of migration criteria would alleviate global inequality of opportunity?}

In order to describe fairer migration regulations, I have to be able to find some criteria that are empirically viable, and sensitive to both the effect on the individual migrants' opportunities, as well as to the effect on the overall opportunities in the country they are moving from and to. In brief, one needs criteria to evaluate whether and to what degree allowing someone to migrate alleviates global inequality of opportunity. In the following, I describe which criteria I believe can fulfill this function, before briefly considering some objections that my model for fairer migration criteria might face. The admission criteria I propose that can manage this must evaluate whether migrating on balance: (1) does not negatively impact the overall level of opportunities in the country of origin through a brain drain effect, (2) positively affects the overall level of opportunities in the receiving country, and (3) distinguishes to which degree a migrant's opportunities will be increased by moving.

This should not be considered an exhaustive list of factors for how migration affects global equality of opportunity. One might for example plausibly argue that it is necessary to consider the effect of migration regulations on global equality of opportunity, as in the overall level of opportunities globally. Some might indeed contend that increased overall migration positively contributes to the global economy and thereby opportunities in general. However, the three factors listed above - effect on country of origin, effect on receiving country, and effect on the opportunities for the individual migrant - seem to be particularly relevant and the factors for which we are most likely to find readily available data. Furthermore, based on the discussion of global metrics of opportunities above, comparisons of potential migrants' opportunities in sending and receiving countries are more likely to be readily available, and empirically sound, than some ideal global metric.

\section{Brain drain, the effects on receiving country and individual migrant}

Moving on to these specific factors, we should first consider how a brain drain effect could negatively affect countries that migrants are moving from. As the phrase implies, this effect is related to the problems countries face when much of the human capital in a country moves abroad. Miller describes this phenomenon, saying,

the ones who have the resources - the savings and the education - that enable them

to do this will be the ones who are already relatively advantaged in their societies 
of origin. [...] It may even turn out that the opportunities of those left behind are reduced in absolute terms, if those leaving are skilled professionals who would otherwise provide education, health services, or competent administration in their home country (2016a: 48).

While there is some disagreement as to the supposed effects more open migration might have on brain drain, this is not the place for that discussion. ${ }^{6}$ However, it is worth considering the negative systemic effects that are caused by brain drains as a result of current migration patterns. As Higgins says: "Large-scale emigration, in fact, initiates a self-perpetuating cycle of underdevelopment in already poor countries, since lost human development prospects both discourage emigrants from returning and encourage more residents to leave" (2013: 67). Furthermore, states are increasingly using highly selective immigration standards, in order to attract the kinds of migrants they wish to admit. Ayelet Shachar points out that "[b]y continually 'retooling and recalibrating' selective skills-based admission avenues to attract the best and brightest, governments engaged in the global race for talent have demonstrated their willingness and their ability to intervene in the market for the highly skilled" (2016: 180).

So, if we wish to avoid the negative effects of brain drain, how will this affect our admission criteria? Intuitively, it implies that when considering migration restrictions, we should weigh the impact the departing migrant has on the overall level of opportunities in the country of origin. A likely result of this could be that we should allow more migration of skilled professionals from countries with more overall opportunities to countries with fewer opportunities than the other way around. This is due to skilled professionals contributing significantly to the overall level of social goods that opportunities rely upon. In order to have a decent health care system, one needs doctors, and in order to have good public education, one needs teachers. When it comes to unskilled migrants, it would seem that fewer restrictions would apply to those moving from less affluent to more affluent societies; one might even argue that according to this first criteria, the less skilled the better. This is due to a large number of unskilled workers often being a strain on social goods such as health care and education, as well as contributing to more competition in the job market. Such evaluations must furthermore be made on the basis of an examination of the opportunities in different countries, as the needs and supply of skilled and unskilled workers will vary from country to country. Different countries also have varying levels of resources with which to provide opportunities for their citizens, and the impact of skilled and unskilled emigration will vary. Some countries will benefit more from emigration, thereby freeing up opportunities in the labor market for the domestic populations, whereas others would suffer more if important institutions were affected by a lack of human capital.

Second, the effect the migrant might have on the receiving country also needs to be taken into account. In relation to this second criterion, similar evaluations to those above must be made, but with the emphasis on the receiving country. Furthermore, in order for the migration restrictions to contribute to global equality of opportunity, one needs to balance the effects on both countries against each other. If the effect of a proposed admission policy greatly benefits either the sending or receiving country to the detriment of the other, it would seem to be problematic. However, if one country benefits greatly, while the other country is only slightly negatively affected, the admission policy would appear to be fairer. 
Third, one also needs to consider the effect migration might have on the opportunities of individual migrants. People have differing levels of opportunity in their countries of origin, due to different factors, such as varying levels of unemployment and access to affordable education. This third criterion will therefore allow us to prioritize individual migrants, where the effect on the first two factors, the sending and receiving countries, is similar. In short, it would enable prioritizing those migrants whose individual opportunities would increase the most by being admitted, while deprioritizing potential migrants who would benefit less.

\section{What would a fairer migration model look like?}

So, how might we envisage a policy that takes the benefits for the migrant, and the receiving and sending countries into account? A popular tool among current types of selective immigration standards is the Australian-style points-based system. A "fairer migration" policy could be modeled on this points system, albeit heavily modified. The Australian system ranks people according to how their skills match the Australian job market, with accountants and mechanics ranked highly, for example (Shachar 2016: 181). Further requirements specify that the migrants should not place a significant burden on the Australian health service, have a criminal record, or pose any threat to the Australian community (Donald 2016). This system evaluates migrants similarly to one of the criteria listed above, namely how beneficial they will be to the country they are moving to.

Conversely, a fairer migration model points system would first consider to what degree the potential migrant's skill is needed in their country of origin, rather than in the receiving country. If the individual was particularly unhealthy or considered a danger to society, on the other hand, this could also cause positive discrimination to be applied, according to this first factor. This person's emigration could be seen as potentially contributing positively to the overall level of opportunities in their country of origin. Following this logic, doctors, university professors, or engineers might be at the back of the queue, if the professions were understaffed in their country of origin. This would broadly allow one to limit potential brain drain effects. Second, one would need to evaluate how much the overall opportunities in the country they are moving to would be improved. If a severe lack of doctors or teachers exists in the receiving country, for example, this would cause these professions to be positively discriminated. This is because of the positive effect they might have on the level of social good, which the opportunities in the receiving country rely upon. This could for example allow for easier movement of skilled workers, such as medical professionals, from the Global North to the Global South than the other way around. This is due their potential impact on overall opportunities being greater in the Global South than the negative impacts of their emigration on countries in the Global North. Third, in order to progress towards global equality of opportunity, people would have to be positively discriminated on the basis of how much their individual opportunities would increase by migrating. In other words, two migrants with the same skill set could be treated differently depending on how much their skills, or lack thereof, benefit them in their country of origin. Faced with many potential migrants, and states that are only willing to admit a limited number, this third criterion would clearly give priority to those individual migrants whose opportunities would increase the most by migrating. 
In order to explicate how one might balance these factors, this fairer migration model can be contrasted with current practices controlling migration. Common migration regulations include: requiring people to earn a certain amount before being eligible to apply for family reunification; needing to show a minimal amount of capital before being accepted on student visas; programs letting people with particular skills receive visas; and programs making it easier to migrate if one is sponsored by an employer in the receiving country. Clearly, these practices can be criticized based on the model presented above. After all, these practices, implicitly or explicitly, merely evaluate a potential migrant on the basis of only one of the three criteria, in this case how much the receiving country might benefit from their arrival.

If we consider some of these specific cases according to the fairer migration model, we can see that some of the evaluations would be significantly different. In particular, it would be more difficult to argue that people should earn a certain amount of money for families to be reunified, if the country they emigrate from does not stand to lose much from their emigration and their individual opportunities are greatly enhanced by migrating. In short, a large positive effect for the migrant and a negligible effect on the country of origin would need to be balanced against a slight downside for the receiving country due to lack of financial means. In the case of student visas, a case might also be made for the students with lesser means being accepted first as it greatly improves their individual opportunities, though this has to be weighed against the receiving state's ability to provide higher education. All in all, the kinds of migration regulations that merely consider the receiving society would have to take account of the impact on the country of origin and on the individual migrant to be justified according to this fairer migration model.

In order to evaluate the viability of such a model, I need to briefly consider some of the objections it might face. However, it is important to initially point out that this fairer migration model does not rule out other considerations of justice, such as the agency of migrants. While it might seem like this system has the potential to be used to justify forced movements in order to alleviate global inequalities, it would clearly be unjust to do so, since this would contravene the fundamental normative consideration of individual agency. Therefore, this system would only apply to people who want to migrate, or who already have and are seeking residency. One might furthermore object to the fairer migration model by arguing that this kind of migration is undesirable, as it could lead to less social unity in the receiving society, and that the migrants I propose should be admitted would be perceived as undeserving. Another detraction might be that this system could create some perverse incentives, whereby individuals would be encouraged to, for example, take less education, thereby limiting their opportunities and increasing their possibilities to migrate. Finally, one might contend that I am ignoring racial and gender discrimination in immigration practices.

While the above concerns would need to be addressed before such a fairer migration model could be implemented, a thorough examination of them is beyond the scope of this article. But it is worth adding that the system outlined above also needs to be balanced against other aims of general immigration policy. It should not overwhelm the receiving society, and there would therefore have to be a limit to the numbers of migrants being admitted. It should also not discriminate against people arbitrarily, on the basis of such factors as race and gender. And while the system could create perverse incentives for some individuals by potentially prioritizing unskilled 
over skilled migrants, it is also doubtful that these incentives will override their motivation to better themselves. Furthermore, a fairer immigration policy should allow for migrants to be properly integrated to ensure the continuing democratic deliberations of the receiving society, and it would somehow have to be broadly accepted among the host population. However, it has to be acknowledged that the latter might be hard to achieve in the current anti-immigration climate that prevails in many Western democracies. In short, any implementation of the fairer migration model would also have to take these objections into consideration.

I should point out that I think it is unlikely that a system such as the one outlined above is likely to be implemented, considering the present situation in which the United Nations refugee agency is finding it difficult to convince countries to resettle even a limited number of refugees. Therefore, rather than delivering a fully-fledged policy proposal, I suggest that these kinds of criteria would be more just according to the demands of global justice. In short, I believe this model would be better able to fulfill the obligations states have to rectify global inequalities that they are responsible for perpetuating. And furthermore, I believe this model would be a far more just system than many current admission criteria that positively discriminate in favor of the wealthy and educated.

\section{Conclusion}

In this article I have considered how one might alter migration regulations in order to contribute to alleviate global inequality of opportunity. I started by considering some of the major objections to the relevance of global inequalities to migration regulations, first by discussing how global inequalities might be compared. While I agree with the criticism that evaluations between various cultural preferences in opportunities can be problematic, I believe that valuable comparisons can be made, in particular in relation to health care, education and the job market. Secondly, I showed why migration is normatively relevant for considerations of global equality of opportunity. In short, as affluent countries both contribute to and benefit from a global trading and financial system that significantly diminishes poorer countries' ability to provide opportunities for their citizens, the affluent countries have a moral obligation to alter this. Furthermore, since these countries do not significantly attempt to alter these underlying conditions, and unfair migration practices are a part of an unfair system, it is pertinent to consider what kind of migration might actually contribute to alleviating inequalities of opportunity. Third, I argued that greater equality of opportunity could in part be met by reevaluating admission policies for migrants. Instead of merely considering how much a country might benefit from receiving a migrant, one should also consider whether, and to what degree, potential migrants might affect the level of opportunity in their country of origin, and to what degree their individual opportunities might be improved by migrating.

I concede that the argument outlined here somewhat oversimplifies affluent states' part in an unjust global trading and financial system. Some states attempt to rectify or maintain this international system to varying degrees. Some differences in opportunities can also be more readily traced back to national priorities than others. Moreover, some countries have more resources than others and are more readily able to admit greater numbers of migrants. I have not shown to what degree various states 
can be seen as responsible for remedying these unjust conditions, as this question is beyond the scope of this article. However, this does not take away from the normative validity of the central argument. In sum, as affluent states benefit greatly from international trading and migration practices to the detriment of less developed states, they are morally obliged to alter their immigration practices in a way that alleviates these inequalities. And while it may not seem feasible to expect states to alter their practices in order to admit migrants whom they do not necessarily benefit from admitting, it is certainly not likely that they will alter their practices if their moral unfairness is not pointed out.

\section{Acknowledgements}

This work has been supported by the Globalizing Minority Rights (GMR) project by The Research Council of Norway under Grant NFR 259017. The publication charges for this article have been funded by a grant from the publication fund of UiT The Arctic University of Norway. Previous versions of the paper have been presented at Polylog's 6th Intercultural Interdisciplinary Colloquium on "Flight and Migration" in Freiburg (Germany), NOS-HS Workshop on Transnational Migration at Linköping University, and Democracy in Context: Contemporary Themes in Ethics and Political Philosophy in Longyearbyen (Norway), organized by Civic Constellation Group, Environmental Research Group (UiT) and Globalizing Minority Rights. Furthermore, I am thankful for comments received at workshops organized by the Pluralism, Democracy and Justice Research Group at UiT. In particular, this manuscript has benefited from valuable suggestions by Annamari Vitikainen, Melina Duarte, Serena Parekh, Yusuf Yuksekdag, Jonas Jakobsen, Sune Lægaard, Andreas Føllesdal, and Nadim Khoury. I would also like to thank the editors and anonymous reviewers for their helpful suggestions and comments, and Thomas Egan for tirelessly proofreading several versions of this paper.

\section{Notes}

${ }^{1}$ This picture is somewhat simplified. Nationalists, such as David Miller (2016a: 7693), also agree that states have extensive duties towards refugees, while cosmopolitans, such as Kieran Oberman (2016: 49-50) and Joseph Carens (2013: 173 179), will agree that states have a justifiable right to limit immigration in exceptional circumstances, for example for health or security reasons. In other words, there is a difference of a degree rather than a strict dichotomy between their positions. However, for the purposes of this paper it makes sense to contrast these positions, as they differ starkly in relation to migration restrictions for voluntary migrants.

${ }^{2}$ The reason for this focus is that there is more of an agreement that global justice should matter when it comes to the treatment of refugees and the forcibly displaced, than in relation to those who migrate voluntarily. Furthermore, it seems quite clear that forcibly moving people around contravenes other basic moral principles, such as individual agency, and it is therefore difficult to see how it can be justified.

${ }^{3}$ The equality of opportunity argument is only one of several arguments Carens puts forward for open borders. He also argues that freedom of movement is an important right in itself, and that it is important to realize other fundamental human rights 
(2013: 225-254). However, as it is the equality of opportunity argument I discuss in this paper, these other arguments will not be considered.

${ }^{4}$ This is quite a broad definition, and there has been an extensive debate on exactly what equality of opportunity consists in. Carens' argument for equality of opportunity relies heavily on Rawls' description in A Theory of Justice, and since then much discussion has revolved around what types of opportunity need to be equalized and what other kinds of social goods equality of opportunity relies upon. My definition is therefore intentionally broad, in order to be able to accommodate various definitions. For various positions on the equality of opportunity globalized, see for example Gillian Brock's Egalitarianism, Ideals, and Cosmopolitan Juistice and Darrel Moellendorf's Equality of Opportunity Globalised?

${ }^{5}$ Scholars do however disagree on the effects migration may have on global differences, and what the potential effects of open borders might be. For example, Kieran Oberman argues that there is substantial evidence that migration in fact reduces poverty (Oberman 2015). Even though it may be the wealthier citizens who migrate, and their families who receive remittances, the money that is then invested and used has broader effects on the sending society. Furthermore, Oberman argues that migration can diffuse knowledge and thereby be beneficial for the sending society, and he shows that an increase in high-skilled emigration can also lead to an increase in the education of highly skilled persons in society in general. While this might be the case, it does not seem to detract from my general line of argument. After all, Oberman also points to the negative effects of brain drain, and that migration restrictions can justifiably be restricted to limit such effects.

${ }^{6}$ See for example Joseph H. Carens The Ethics of Immigration pp. 183-184.

\section{References}

Brock, G. (2005, March). Egalitarianism, ideals, and cosmopolitan justice. In The Philosophical Forum (Vol. 36, No. 1, pp. 1-30). Oxford, UK and Malden, USA: Blackwell Publishing Ltd. https://doi.org/10.1111/j.1467-9191.2005.00186.x

Carens, J. H. (2013). The Ethics of Immigration. Oxford: Oxford University Press.

Donald, A. (2016). Immigration Points-Based Systems Compared. BBC News, 1. Retrieved November 2017, http://www.bbc.com/news/uk-politics-29594642.

Hickel, J. (2014). Aid in reverse: how poor countries develop rich countries. The Guardian. Retrieved November 2017, https://www.theguardian.com/globaldevelopment-professionals-network/2017/jan/14/aid-in-reverse-how-poorcountries-develop-rich-countries?CMP=Share_iOSApp_Other.

Higgins, P. (2013). Immigration justice. Edinburgh University Press. https://doi.org/ 10.3366/edinburgh/9780748670260.001.0001

Kollar, E. (2017). Global equality of opportunity and self-determination in the context of immigration. Critical Review of International Social and Political Philosophy, 20(6), 726-735. https://doi.org/10.1080/13698230.2016.1231753

Miller, D. (2016). Strangers in our midst. Harvard University Press. https://doi.org/ $10.4159 / 9780674969827$

Miller, D. (2017). Migration and justice: a reply to my critics. Critical Review of International Social and Political Philosophy, 20(6), 763-773. https://doi.org/ $10.1080 / 13698230.2016 .1231847$ 
Moellendorf, D. (2006). Equality of opportunity globalized?. Canadian Journal of Law \& Jurisprudence, 19(2), 301-318. https://doi.org/10.1017/S0841820900004124

Oberman, K. (2015). Poverty and immigration policy. American Political Science Review, 109(2), 239-251. https://doi.org/10.1093/acprof:oso/9780199676606.003.0003

Oberman, K. (2016). Immigration as a human right. Migration in political theory: The ethics of movement and membership, 109, 32-56.

Rawls, J. (1971). A Theory of Justice. Harvard University Press, Cambridge.

Shachar, A. (2016). "The Brave New World of Stratified Mobility", in Fine, S., \& Ypi, L. (Eds.). (2016). Migration in political theory: the ethics of movement and membership. Oxford University Press. 175-201.

Pogge, T. (1997). Migration and poverty. In Citizenship and exclusion (pp. 12-27).

Palgrave Macmillan, London. https://doi.org/10.1057/9780230374591_2

Pogge, T.W. (ed), (2001). Global Justice, Blackwell, Oxford. 